ACCEPTED For PUblication in ApJ

Preprint typeset using $\mathrm{LAT}_{\mathrm{E}} \mathrm{X}$ style emulateapj v. 5/2/11

\title{
THE COSMIC EVOLUTION OF FAINT SATELLITE GALAXIES AS A TEST OF GALAXY FORMATION AND THE NATURE OF DARK MATTER
}

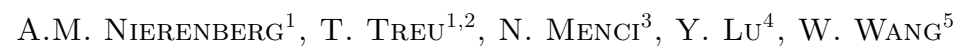 \\ Accepted for publication in ApJ
}

\begin{abstract}
The standard cosmological model based on cold dark matter (CDM) predicts a large number of subhalos for each galaxy-size halo. Matching the subhalos to the observed properties of luminous satellites of galaxies in the local universe poses a significant challenge to our understanding of the astrophysics of galaxy formation. We show that the cosmic evolution and host mass dependence of the luminosity function of satellites provides a powerful new diagnostic to disentangle astrophysical effects from variations in the underlying dark matter mass function. We illustrate this by comparing recent observations of satellites between redshifts $0.1<z<0.8$ based on Hubble Space Telescope images, with predictions from of three different state-of-the art semi-analytic models applied to CDM power spectra, with one model also applied to a Warm Dark Matter (WDM) spectrum. We find that even though CDM models provide a reasonable fit to the local luminosity function of satellites around galaxies comparable to the Milky Way, they do not reproduce the data as well for different redshifts and host galaxy stellar masses, indicating that further improvements in the description of star formation are likely needed. The WDM model matches the observed mass dependence and redshift evolution of satellite galaxies more closely, indicating that a modification of the underlying power spectrum may offer an alternative solution to this tension. We conclude by presenting predictions for the color distribution of satellite galaxies to demonstrate how future observations will be able to further distinguish between these models and help constrain baryonic and non-baryonic physics.
\end{abstract}

Subject headings: galaxies: halos — galaxies: structure — galaxies:dwarf - dark matter

\section{INTRODUCTION}

The standard model based on dark energy and Cold Dark Matter (CDM) is successful at describing the large-scale ( $>\mathrm{Mpc}$ ) structure of the universe (e.g. Komatsu et al. 2011). In this standard model, galaxies and clusters of galaxies grow hierarchically within dark matter halos (e.g., Springel et al. 2006).

At the scales typical of galaxies $(\sim \mathrm{kpc})$, the model faces a number of observational challenges. A major source of tension between observation and the standard model is the so-called "missing satellite problem" (Klypin et al. 1999; Moore et al. 1999). At large scales, a simple ranked matching of simulated subhalos to observed luminous structure predicts clustering behavior consistent with that of the observed luminous structure (Conroy \& Wechsler 2009; Behroozi et al.|2010). At smaller scales however, this simple matching scheme breaks down. For instance, CDM simulations predict that the Milky Way (MW) should have approximately 10 subhalos with rotation velocities greater than Fornax, while only 3 satellites that are more luminous are observed (Boylan-Kolchin et al. 2012; Strigari \& Wechsler 2012). The discrepancy between the predicted number

\footnotetext{
${ }^{1}$ Department of Physics, University of California, Santa Barbara, CA 93106, USA; amn01@physics.ucsb.edu

2 Packard Fellow

3 NAF - Osservatorio Astronomico di Roma, via di Frascati 33, I-00040 Monteporzio, Italy

${ }^{4}$ Kavli Institute for Particle Astrophysics and Cosmology, Stanford CA, 94309

${ }^{5}$ Key Laboratory for Research in Galaxies and Cosmology of Chinese Academy of Sciences, Max-Panck-Institute Partner Group, Shanghai Astronomical Observatory, Nandan Road 80, Shanghai 200030, China
}

of subhalos and the observed number of luminous satellites becomes more dramatic at even lower halo masses (Strigari et al. 2007). While the measurement of low luminosity satellites may be biased in the Milky Way due to disk obscuration (Tollerud et al. 2008), the lack of low mass galaxies extends to the field as well, where Papastergis et al. (2011) measured a factor of 8 fewer galaxies with velocity widths of $\sim 50 \mathrm{~km} / \mathrm{s}$ than predicted by current $\Lambda$ CDM simulations. Furthermore, recent surveys have found that there is not a significant population of optically dark, gas rich galaxies, (Doyle et al. 2005), indicating that if these very low mass halos exist, they do not contain significant amounts of gas or stars.

The discrepancy may be due to a variety of factors. The complex physics of star formation in low mass halos makes matching simulated halos to observed satellite galaxies difficult. Significant progress has been made in understanding the complex processes which affect star formation in low mass halos (Kravtsov 2010). In particular, numerous studies have focused on studying how both sub-galactic effects such as supernovae feedback and stellar winds, and super-galactic effects such as UV heating from reionization, and tidal and ram pressure stripping by the central galaxy can suppress star formation and thereby produce the present day satellite luminosity function (e.g. Bullock et al.|2000; Benson et al.|2002; Somerville 2002; Kravtsov et al. 2004; Kaufmann et al. 2008; Macciò 2010; Springel 2010).

In addition to suppressing star formation, thereby making subhalos undetectable, several studies have examined whether supernovae feedback can significantly flatten the central regions of dark matter density profiles. This would potentially explain the discrep- 
ancy between the predicted and observed central velocities of $\Lambda \mathrm{CDM}$ subhalos around the Milky Way (Boylan-Kolchin et al. 2012), by lowering the subhalo central velocities, thereby making them appear less massive. Simulations with baryons have produced conflicting predictions as to whether supernovae can (Governato et al. 2012; Tevssier et al. 2013) or cannot (Garrison-Kimmel et al. 2013) significantly alter the kinematics in the central regions of dark matter halos.

The issue of the low central velocities of Milky Way subhalos is further complicated by the fact that the $\Lambda \mathrm{CDM}$ prediction for the subhalo circular velocity function depends sensitively on the virial mass of the Milky Way halo. Wang et al. (2012) showed that taking into account the uncertainty on the measured Milky Way virial mass, the observed subhalo circular velocity function was consistent with that observed in ensemble simulations of dark matter halos. Purcell \& Zentner (2012) further showed that even simulated halos with masses corresponding to the observational mean have subhalo populations consistent with the Milky Way a significant fraction $(\sim 10 \%)$ of the time.

These studies demonstrate that it is essential to study the statistical properties of satellite galaxies in observation and simulations for a large sample of host galaxies, in order to determine whether apparent discrepancies are driven by the stochastic nature of galaxy formation, or observational uncertainty, rather than poorly modeled physical processes.

Furthermore, as the baryonic processes which affect the subhalo population occur over cosmological time scales, ideally the predicted effects would be compared with observations of the satellite population over as much of the history of the universe as possible.

If the solution to this problem is astrophysical in nature, i.e. low mass halos exist but do not form stars and are therefore undetected by traditional astronomical observations, one needs to turn to other methods to verify their existence. This line of reasoning has motivated searches for satellites halos based on properties that are independent of their stellar content, such as their gravitational lensing effect (e.g. Mao \& Schneider 1998; Metcalf \& Madau 2001; Dalal \& Kochanek 2002; Amara et al. 2006; Keeton \& Moustakas 2009; Treu 2010; Vegetti et al. 2012), or on their expected DM annihilation signal (Kuhlen et al. 2008; Porter et al. 2011; Strigari 2012; Abazajian \& Kaplinghat 2012; Fadely \& Keeton 2012), or their influence of tidal streams in the Milky Way (Carlberg et al. 2012). Direct detection of dark subhalos would be a stunning confirmation of the standard model.

However, this is not the only possible solution to the missing satellite problem. Warm Dark Matter (WDM) (e.g. Colombi et al. 1996, and references therein) is an interesting alternative to CDM, potentially offering an elegant astroparticle solution to the missing satellite problem (Lovell et al. 2012). In WDM scenarios, small-scale structure is suppressed relative to CDM. There are a variety of mechanisms which can achieve this, for instance either by reducing the mass of the dark matter particle for a thermal relic (e.g Steffen 2006) or by introducing non-thermal particles such as sterile neutrinos produced from oscillations of active neutrinos (e.g. Olive \& Turner 1982; Shi \& Fuller 1999;
Abazajian et al. 2001; Dolgov \& Hansen 2002). The suppression of small-scale structure in turn affects the full merger history of a halo and its subhalos, which recent cosmological simulations have begun to study in detail (Menci et al. 2012; Lovell et al. 2012; Kamada et al. 2013; Polisensky \& Ricotti 2011; Bode et al. 2001; Gottloeber et al. 2010; de Vega \& Sanchez 2012).

Clearly the astrophysical and astroparticle solutions to the missing satellite problem are not mutually exclusive, and progress on both fronts may be needed to reconcile the model with the data. In fact, a proper observational test of the WDM model predictions for the number of satellite galaxies requires an accurate description of the astrophysical effects related to star formation.

One difficulty in studying this issue, is that is impossible to distinguish the effects of varying baryonic physics from suppressing the subhalo mass function using only low redshift measurements of the satellite or field luminosity function. However, with multiple observables, one may begin to disentangle the effects. For instance, Kang et al. (2013), showed that if the WDM particle is too light, one cannot simultaneously reproduce the TullyFisher relation and the field luminosity function.

In this paper we present a new test of three semianalytic galaxy formation models, with one model implemented for both a Warm and Cold Dark Matter substructure mass function, in order to show how variations in the halo mass function produce different predictions from variations in baryonic physics. We compare the predictions from these models with the observed abundance of satellite galaxies as a function of host galaxy mass and cosmic time.

This test is made possible by two recent developments. On the observational side, the implementation of powerful statistical tools to detect and count satellites in deep archival Hubble Space Telescope images provides data to compare with model predictions (Nierenberg et al. 2011, 2012). On the theoretical side, advances in computational methods have allowed for cosmological simulations with unprecedented volume and resolution. In this work we focus on predictions from four independent cosmological simulations, which have semi-analytic models applied to dark matter merging trees, which we describe in more detail below.

The paper is organized as follows: In $₫ 2$ we describe our observations of the satellite luminosity function. In $\$ 3$ we summarize the key aspects of the theoretical models. In 8 , we compare the observations with the theoretical predictions. In $\$ 5$ we compare a few of the main properties of the models and present new predictions for the distribution of colors of satellite galaxies across cosmic time. In 6 we compare the model results with other observations taken from the literature. Finally in $\$ 7$ we conclude with a discussion and summary of the results.

\section{OBSERVATIONS}

Measurements of the satellite population were obtained by Nierenberg et al. (2012), using ACS F814W imaging of the COSMOS field, where a full description of the analysis is given.

For convenience of the reader, the detection technique is illustrated in Figure 2. First, the depth and high angular resolution of Hubble images was used to identify satellites as much as a thousand times fainter than their host 
galaxies, and out to redshift $z=0.8$. After adding simulated satellites near bright central galaxies, and testing object recovery and accuracy, the analysis was restricted to objects with $\mathrm{F} 814<25$ mag $\mathrm{AB}$, in order to ensure completeness.

Using a single band of photometry, Nierenberg et al. (2012) model the number density of objects near the chosen host galaxies as a combination of a uniform density of background/foreground objects, in addition to a population of satellite galaxies which have a number density which increases radially as a power law near the host galaxies (e.g. Chen 2008; Watson et al. 2012. Nierenberg et al. 2011, 2012). The power of the radial distribution as well as the number of satellites within a fixed magnitude offset from the host magnitude are simultaneously inferred to yield a cumulative luminosity function.

Nierenberg et al. (2012) showed that the inferred satellite luminosity function for low mass host galaxies is fully consistent with the luminosity function of Milky Way satellites, and the satellites of other similar mass galaxies at low redshift (Strigari \& Wechsler 2012; Liu et al. 2011; Guo et al. 2011a). Taking into account the differences between global background subtraction rather than the locally estimated background used in our measurement, which can cause a factor of two increase in the inferred number of satellites due to correlated structure around massive host galaxies, our results are also consistent with Wang \& White (2012) at low redshifts and for the most massive satellites $\left(\log \left[L_{s} / L_{h}\right]>-1\right)$, our results are consistent with those of (Newman et al. 2012) at higher redshifts.

As we will show below, these new constraints on host mass and redshift dependence of the satellite luminosity function provide distinguishing power between the physical effects of varying the halo mass function and star formation physics on the satellite luminosity function.

\section{THEORETICAL MODELS}

We study three different semi-analytic models implemented in three different cosmological Cold Dark Matter simulations. In addition, we apply one of the semianalytic models to a Warm Dark Matter cosmology. This section provides a description of both the Cold and Warm Dark Matter simulations and their models for galaxy formation.

\subsection{Cold Dark Matter Models}

When studying the satellite luminosity function, it is extremely useful to compare multiple cosmological simulations along with different star formation parameters in order to understand the range of theoretical predictions given different assumptions. In this work we focus on three Cold Dark Matter cosmological simulations, each with its own semi-analytic model for galaxy. The simulation parameters governing star formation and the underlying dark matter mass function were fully specified prior to performing this test and are the same as in previous papers, in which a more complete description can be found (Menci et al. 2012; Lu et al. 2012; Guo et al. 2011b). Below we briefly summarize the relevant aspects of these simulations.

\subsubsection{Menci et al. 2012}

The semi-analytic models are described in detail by Menci et al. (2012). In brief, the backbone of the models are computations of dark matter merging histories that can resolve halos down to masses of $10^{5} \mathrm{M}_{\odot}$, allowing for an extremely accurate characterization of the survival and merger histories of satellites. This is essential for studying the faint end of the satellite luminosity function. The models predict mass distributions of halos, and the merging rates of DM halos and subhalos based on an input power spectrum, and generate luminosities for these halos based on a set of star formation prescriptions.

These galaxy formation prescriptions connect the complex baryon physics of star formation to the dynamical evolution of the DM haloes. For each subhalo hosting a galaxy, the model predicts radiative gas cooling, ensuing star formation and associated feedback from Supernovae (SNae) events. Cooled gas settles into a rotationally supported disk with radius and rotational velocity related to the DM mass of the subhalo. This gas gradually condenses into stars at a rate consistent with the observed Kennicut-Schmidt law (Kennicutt 1998). SNae return part of the cooled gas to the hot gas phase at the virial temperature of the halo. Star formation is also triggered by galaxy-galaxy interaction-driven starbursts, which induce gas accretion onto the central supermassive Black Holes.

It is important to note that the set of parameters used in these models is much smaller than the number of observables they are simultaneously consistent with. In brief, the star formation parameters are tuned to simultaneously match the field luminosity function in multiple bands from redshifts 0 to 6 , the Tully-Fisher relation (Tully \& Fisher 1977), the stellar mass function of field galaxies from between redshifts 0 and 4 , the stellar massstar formation relation to a redshift of 2 , the colors and color magnitude relations of both field and cluster galaxies in addition to the AGN luminosity function.

Nierenberg et al. (2012) required host galaxies to be outside of $\mathrm{R}_{200}$ of any galaxy with a higher stellar mass, where $R_{200}$ was estimated using the formula from Dutton et al. (2010). Ideally this selection would be duplicated when comparing with the simulation, however this is not possible for the Menci et al. (2012) models, as they do not contain spatial information. This should not significantly affect our results, as comparisons with the Millennium and Bolshoi N-Body simulations (described below) show that the Nierenberg et al. (2012) central isolation criteria are efficient at returning central host galaxies $(\sim 90 \%)$, and that the use of the matching selection criteria in these cases did not significantly alter the inferred predicted number of satellites per host, relative to simply using the simulation selection of central galaxies.

\subsubsection{Guo et al. 2011}

The semi-analytic model of Guo et al. (2011b) is based on two very large dark matter simulations, the Millennium Simulation (MS; Springel et al. 2005) and the Millennium-II Simulation (MS-II; Bovlan-Kolchin et al. 2009). The box size of the MS is $500 h^{-1} \mathrm{Mpc}$ and its merger trees are complete for subhaloes above a mass limit of $1.7 \times 10^{10} h^{-1} \mathrm{M}_{\odot}$. The MS-II follows a cube of side $100 h^{-1} \mathrm{Mpc}$, but with 125 times better mass resolution than the MS (subhalo masses greater than 


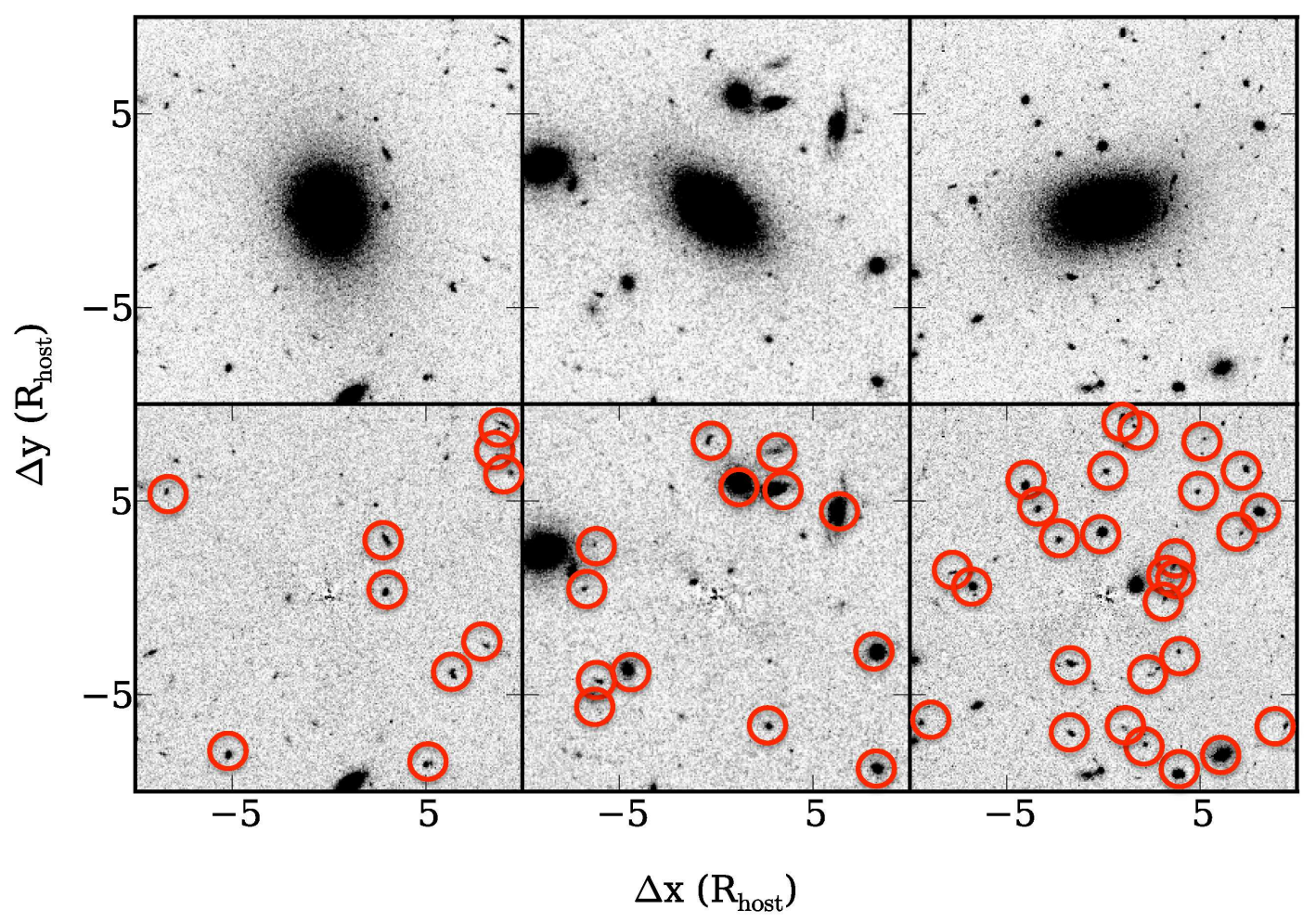

FIG. 1.- Demonstration of our image processing technique. The upper panels show the original Hubble Space Telescope images centered on main galaxies, while lower panels show the host-subtracted images. Red circles show objects we detect near the hosts. While we study the satellite population out to much larger radii (about 5 times larger than the images), the host subtraction in the central region is essential for allowing an accurate characterization of the satellite spatial distribution, which in turn allows us to isolate the properties of the satellite population. Some objects that are too close to the edges, or which are below our detection threshold are not circled. Objects very close to the centers of the host galaxies are excluded as the host subtraction becomes inaccurate in the inner regions.

$\left.1.4 \times 10^{8} h^{-1} \mathrm{M}_{\odot}\right)$. Both simulations adopt the WMAP1based $\Lambda$ CDM cosmology (Spergel et al. 2003) with parameters $h=0.73, \Omega_{m}=0.25, \Omega_{\Lambda}=0.75, n=1$ and $\sigma_{8}=0.9$. Due to the lower resolution of the MS, Wang \& White (2012) found that the luminosity functions of satellites flattens for satellites with $M_{r}>-18$. For brighter satellites with $M_{r}<-18$, the simulations are consistent. Since satellite luminosity functions will be measured to about three-orders of magnitude fainter than central primaries in this paper, we will focus on the semi-analytic model implemented on MS-II hereafter.

In general, the galaxy evolution model of Guo et al. $(2011 \mathrm{~b})$ is based on those developed by Springel et al. (2005); Croton et al. (2006); De Lucia \& Blaizot (2007). The model includes a few main modifications such as the different definition of satellite galaxies, the gradual stripping and disruption of satellites, a mass-dependent model of supernova feedback, a modified model for reionization and a more realistic treatment of the growth of stellar and gaseous disks. Free parameters of these mod- els by (Guo et al. 2011b) were determined to give close predictions to the abundance and clustering of low redshift galaxies, as inferred from SDSS, and are functions of their stellar mass, luminosity and color.

There are two types of satellites in the simulation: $i$ ) those with an associated dark matter subhalo (type-1) and $i i)$ those whose dark subhalo has fallen below the resolution limit of the simulation (type-2). For the latter, the position and velocity of the orphan galaxy is given by those of their most bound particle. Type- 2 satellites are removed from the galaxy catalogues when one of these two conditions is fulfilled: 1) the time passed from the disruption of the subhalo is longer than their estimated dynamical-friction timescale, or 2) the integrated tidal forces from the host halo exceed the binding energy of the galaxy.

Here we use the data downloaded from http://www.mpagarching.mpg.de/millennium for registered users. We project the simulation box in three orthogonal directions (along their $x, y$ and $z$ axes). In 
each projection we assign each galaxy a redshift based on its "line-of-sight" distance and peculiar velocity. We select isolated primaries using criteria which are directly analogous to those used by Nierenberg et al. (2012). Satellites are defined to be all companion galaxies whose distances to the isolated primaries are smaller than the halo virial radius $\left(\mathrm{R}_{200}\right)$. To directly compare results based on COSMOS, the real $\mathrm{R}_{\text {vir }}$ provided in the database is ignored, and instead we used the empirical formula of Dutton et al. (2010) to estimate $\mathrm{M}_{\mathrm{vir}}$ and $R_{200}$ from stellar masses of galaxies.

\subsubsection{Lu et al. 2012}

The baryonic processes implemented in this semianalytic model are described in Lu et al. (2011, 2012). Different from the previously published versions, this version of the model is applied on a set of halo merger trees extracted from a large cosmological $N$-body simulation, the Bolshoi simulation (Klypin et al. 2011), which has a box size $250 h^{-1} \mathrm{Mpc}$ on a side. The simulation adopted a cosmology favored by WMAP7 data (Jarosik et al.|2011) and WMAP5 data (Dunkley et al. 2009; Komatsu et al. 2009 ) with parameters with $\Omega_{\mathrm{m}, \mathrm{o}}=0.27, \Omega_{\Lambda, o}=0.73$, $\Omega_{\mathrm{b}, \mathrm{o}}=0.044, h=0.70, n=0.95$ and $\sigma_{8}=0.82$. The mass resolution of the simulation is $1.35 \times 10^{8} h^{-1} \mathrm{M}_{\odot}$, which allows us to track halos and subhalos with mass $\sim 7 \times 10^{9} h^{-1} \mathrm{M}_{\odot}$. Dark matter halos and subhalos are identified with the Rockstar halo finder (Behroozi et al. 2012) based on adaptive hierarchical refinement in phasespace.

As with the two other models in this work, the semianalytic model follows the dark matter merger tree and calculates the rates of gas cooling, star formation, outflow induced by star formation feedback, and galaxy-galaxy mergers. The kinematics of satellite galaxies is followed by using subhalo information from the simulation whenever the subhalo is resolved. When a halo becomes a subhalo, we instantaneously strip the hot gas associated with the halo, while the stellar mass and cold gas mass remain intact.

When the subhalo is no longer resolved in the simulation, the model applies dynamical friction (Binney \& Tremaine 1987) to estimate when the satellite has merged into the central galaxy, and it assumes that the tidal stripping is strong enough to also strip the stellar mass and cold gas mass. At this point, the entire cold gas disk is stripped and is mixed into the hot gas of the host primary halo. Starting from that time, a fraction of stellar mass is tidally stripped per orbital timescale. The efficiency of the tidal stripping is controlled by a parameter, which is tuned to yield $30 \%$ of the stellar mass is stripped in every orbital timescale to match the conditional stellar mass function of local galaxies.

Other parameters governing star formation and feedback are tuned using an MCMC optimization to match the local galaxy stellar mass function (Moustakas et al 2013). The model is guaranteed to produce a global galaxy stellar mass function which provides the best possible match to the data between $10^{9}$ and $10^{12} \mathrm{M}_{\odot}$ at redshift zero, within the observational uncertainty and given the chosen model parametrization.

To compare with observation, hosts were selected using the same isolation criteria as used in Nierenberg et al.
(2012), with hosts required to be not within $R_{200}$ of a host with higher stellar mass, where $R_{200}$ is estimated from Dutton et al. (2010), using the relationship for early-type hosts. The satellite luminosity function was measured within this region, rather within $R_{v i r}$ as given by the simulation.

\subsection{Menci Warm Dark Matter Model}

Warm Dark Matter has been proposed as a means of suppressing the satellite luminosity function by reducing the number of low mass dark matter halos, suppressing power with respect to CDM below a certain cutoff-scale.

In this work, we use WDM merger trees from Menci et al. (2012) which are based on a cutoff scale of $\sim 1 \mathrm{Mpc}$, corresponding to a $\sim 0.75 \mathrm{keV} / \mathrm{c}^{2}$ thermal relic, which suppresses the power spectrum on sub-Mpc scales and has behavior equivalent to that of a $\sim 3.4$ $\mathrm{keV} / \mathrm{c}^{2}$ sterile neutrino. We chose this mass to be low enough to affect satellite-scale structure while still agreeing with limits from observations of large-scale and local group structure which constrain the particle mass to be larger than $0.6 \mathrm{keV} / c^{2}$ for a thermal relic and 2.5 $\mathrm{keV} / \mathrm{c}^{2}$ for a sterile neutrino DM particle (Viel et al. 2009; Bovarskv et al. 2009; Polisensky \& Ricotti 2011; Kang et al. 2013).

The fiducial mass function from Menci et al. (2012) was based on an Extended Press Schechter formalism (EPS, Bond et al. 1991). In our predictions, we consider the effects of a complete suppression of progenitors with masses below that corresponding to the freestreaming scale; this maximizes the possible effects of different window functions and collapse thresholds in the WDM merging trees (see Benson et al. 2013). We include the possible range of model predictions under different assumptions for the building up of merging trees in the uncertainty regions in Figure 2.

The mass function from Menci et al. (2012) is based on on the Extended Press Schechter formalism (EPS, Bond et al. 1991), which is modified to take into account the suppression of structure below the free-streaming scale (see, e.g. Menci et al. 2012; Benson et al. 2013). The uncertainties associated with this modification are reflected in the width of the prediction for the final WDM satellite luminosity function.

Our goal in this comparison was to explore how the effects of varying the power spectrum compare with the effects of varying semi-analytic star formation parameters. To achieve this, the same semi-analytic model of star formation is used for the WDM models as was applied to the CDM merging trees in the Menci CDM model. The CDM semi-analytic model was selected to provide a good match to the field color distribution. Menci et al. (2012) show that this model provides a good fit to the field luminosity function when applied to a WDM power spectrum.

\section{RESULTS}

The improvements in observations and the implementation of semi-analytic models in cosmological scale simulations allows a comparison between the observed and predicted number of satellites as a function of host galaxy mass and cosmic time for the first time, thus allowing for significantly more discriminatory power than tests based only on the MW or the local volume. 


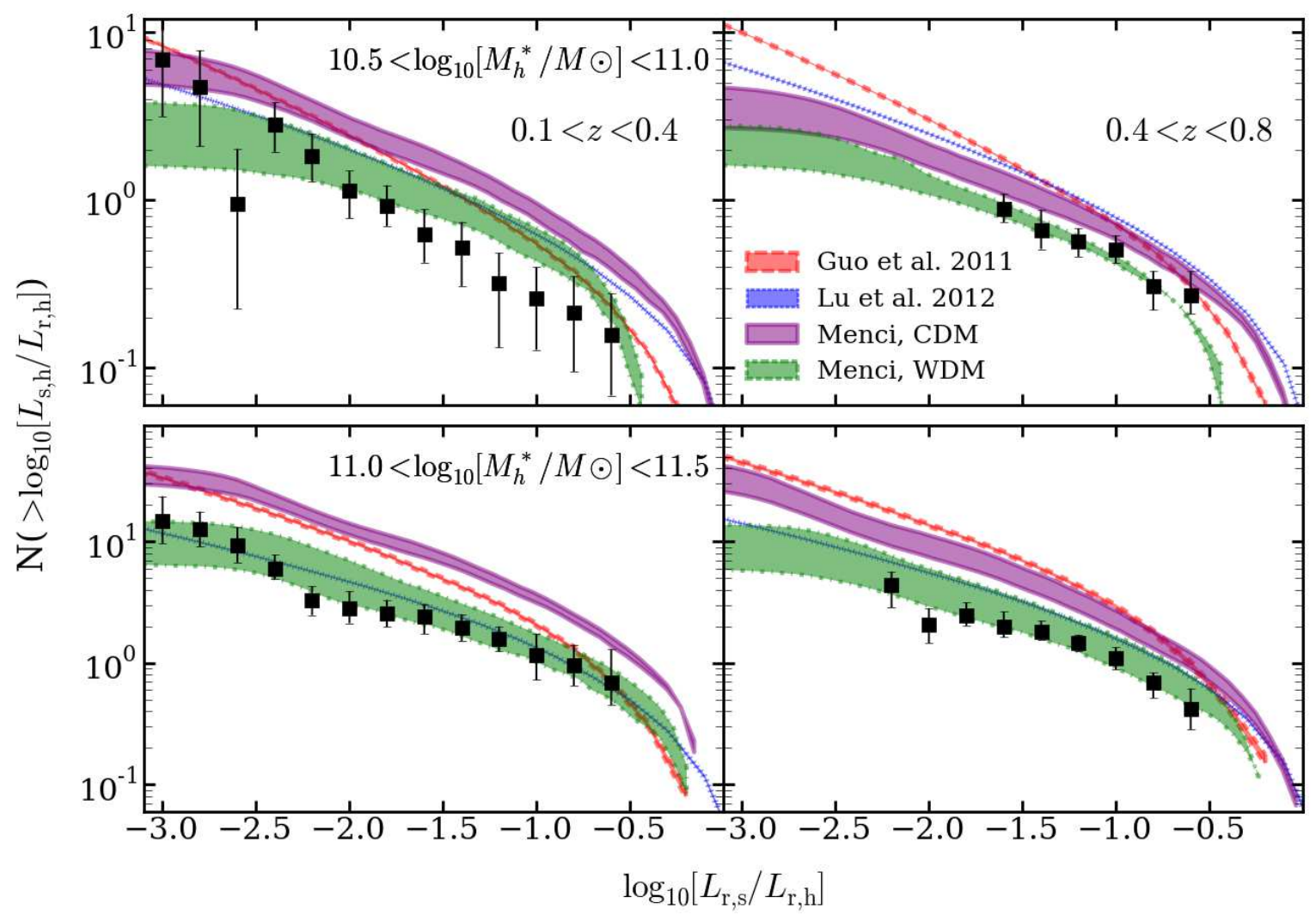

FIG. 2.- Comparison of CDM and WDM model predictions to observed number of satellites over a range of redshift and host stellar masses. The purple, blue and red curves represent predictions from three CDM based simulations with separate semi-analytic models for star formation. The gray curve shows the prediction for one WDM simulation with star formation parameters identical to those used in Menci, CDM prediction. For the Lu and Guo models, the line widths represent the scatter in the mean value across the simulation boxes. For the Menci CDM model, the shaded region represents the range of model predictions possible given the observational uncertainty in the selection of host halo masses. For the Menci WDM model, the shaded region also accounts for the uncertainties in the suppression of progenitors below the free-streaming scale (see 3.2) The points with vertical error bars are measurements (Nierenberg et al. 2012). The top panels show the comparison for lower mass hosts at lower (left) and higher redshift (right), while the lower panels are the equivalent for higher mass hosts.

The results of the comparison are presented in Figure 2, where we plot the observed number of satellites as a function of the ratio between host and satellite luminosities in SDSS-r, along with the WDM and CDM model predictions. Among the CDM model predictions, two main trends are evident. First, no one CDM prediction precisely matches the observation at all redshift and stellar mass intervals. This highlights the importance of using data from a range of redshift and stellar mass when tuning the parameters of semi-analytic models. All three models show qualitatively similar behavior with a strong dependence on the number of satellites per host on the host galaxy mass. Taking into account the covariance between data points (calculated by bootstrap resampling), the generalized chi-squared between the models and the data is 529, 653 and 105 for the Menci, Guo and Lu models respectively for 41 degrees of freedom.

The Menci WDM model shows distinct behavior in comparison with the CDM models. Notably, it predicts weaker host mass dependence and less redshift evolution than predicted by any of the CDM models. Of the four models it provides the best agreement with the data with a chi-squared of 56.

One of the most interesting results of the above comparison is the significant difference in the predicted satellite luminosity functions, even among the three CDM models. In the following section, we perform a detailed comparison of some of the properties of the four models in order to explore the cause of these differences.

\section{COMPARISON OF SIMULATION PROPERTIES}

In the previous section we found significant differences in the model predictions for the satellite luminosity function. As discussed in Section 3 numerous physical processes contribute to the final predicted luminosity function. In this section, we compare key aspects of the models in order to elucidate which model assumptions drive the predicted differences. We first compare the subhalo mass function in the models, before the effects of tidal stripping take effect, for all subhalos regardless of final luminosity. We then show the stellar mass to halo mass relationship for hosts and satellites in the luminosity range considered in this work, before the subhalos have undergone tidal stripping. We conclude by comparing the predicted satellite colors to show the differences in star 
formation histories.

\subsection{Unstripped subhalo mass function}

The four simulations all assumed slightly different cosmologies and had different mass resolutions, so it is important to consider how much the differences in the predicted results are due to these effects. In Figure 3 we plot the mass function of subhalos in a mass range representative of the hosts and subhaloes from Figure 2 Note that this mass function is purely based on dark matter mass, and does not directly correspond to the satellite galaxies in Figure 2, as there is no luminosity selection.

The Guo, Menci, and Lu CDM subhalo mass functions are extremely similar, while the WDM model shows significant truncation for subhalos with $\log \left[M_{\text {sub }} / M_{\text {host }}\right]<$ -1 for the less massive hosts and $\log \left[M_{s u b} / M_{\text {host }}\right]<$ -1.5 for the more massive hosts, and predicts similar behavior to other WDM models with sub-Mpc cutoff scales (e.g. Colín et al. 2000; Smith \& Markovic 2011; Kamada et al. 2013).

\subsection{The halo mass to stellar mass relationship}

One of the most important physical processes relied on in this comparison between simulation and observation, is the relationship between host halo mass and stellar mass, because the number of subhalos around a given host galaxy is strongly dependent on the halo mass of the host galaxy (see e.g. Busha et al. 2011), particularly in the case of CDM. Furthermore, as we always consider the quantity $\log _{10}\left[L_{s} / L_{h}\right]>-3$, it is important to distinguish whether differences in the models are caused by differences in $L_{s}$ or in $L_{h}$.

In Figure 4 we plot the halo to stellar mass relationship for the four models, in addition to the observed relationship from Dutton et al. (2010), which was used to estimate $R_{200}$ for the host galaxies in the observations. For bright galaxies with stellar masses greater than $10^{10.5} \mathrm{M}_{\odot}$, the models are all very similar to each other and to the observed relationship, within the large scatter. Thus we conclude that the differences in amplitude in the predictions for the satellite luminosity function are not driven primarily by differences in the halo to stellar mass relationship for host galaxies.

Towards the faint end, all models are very similar within the scatter, although the Menci models show a marginally higher dark matter mass at fixed stellar mass. As we show in the following section, the Menci satellite colors tend to be bluer than the Guo and Lu colors. From (Bell et al. 2003), Table 7, the bluer colors of the Menci model, correspond to an $\mathrm{r}$ band mass-to-light ratio which is lower by roughly $0.1-0.2$ dex on average than for the Guo and Lu models. Thus, as the final luminosity functions are similar, it is expected that the Menci models would produce galaxies with on average slightly lower stellar masses for fixed halo mass.

\subsection{Satellite galaxy colors}

The intrinsic colors of satellite galaxies are dependent on the star formation history. As discussed above, this is determined by the metallicity, feedback and UV heating, in addition to environmental effects as the satellites enter the influence of the host galaxy halo. All three models used the same stellar population synthesis models from (Bruzual \& Charlot 2003), thus satellite galaxy colors provide an important means of distinguishing between different physical models for the suppression of star formation in low mass halos. Two models which produce similar luminosity functions may rely on very different star formation prescriptions, which will result in different color distributions. In Figure 5, we plot the predicted distribution of rest-frame $\mathrm{u}$-i colors for satellites with $\log _{10}\left[L_{s} / L_{h}\right]>-3$.

In order to summarize these predictions, we fit each of the distributions to a skewed normal distribution (Azzalini 1985) where the probability of having color $c$ is given by:

$$
P(c) \propto e^{\frac{\left(c-c_{o}\right)^{2}}{2 \sigma^{2}}}\left(1+\operatorname{erf}\left(\frac{a\left(c-c_{o}\right)}{\sqrt{2} \sigma}\right)\right)
$$

Here $c_{o}$ and $\sigma$ are the usual mean and standard deviations of a normal distribution while the parameter $a$ describes the skewness. The best fit values for these parameters are listed in Table $1^{6}$. The parametrization of the distributions as being skewed normal is not physically motivated, but rather intended to facilitate future comparison between observation and these predictions.

The models all predict significantly different color distributions for the satellite galaxies, with the exception of the Menci CDM and WDM models, for which the same star formation parameters were used. We highlight the fact that although the luminosity function predicted by the Menci WDM and Lu CDM models are similar within some redshift and host stellar mass ranges, the color distributions are very different. This is due to the fact that in the $\mathrm{Lu}$ CDM model, the faint end of the luminosity function is suppressed mostly by the effects of feedback and ram pressure stripping and heating of the gas by the host halo, while in the Menci WDM model, the luminosity function is suppressed by the lack of low mass subhalos, as expected.

In order to facilitate comparison with observation, it is important to also consider the effects of dust extinction. In Figure 6. we show the prediction for the rest-frame colors of satellite galaxies with dust. The Menci models behave very differently with the addition of dust, relative to the Guo and Lu models, with the Menci color distributions becoming much wider and significantly redder while the Guo and Lu models develop a longer redward tail, without significant other alteration. The secondary peaks in the Menci model become more prominent with the addition of dust, so we fit the Menci models with the sum of two skewed normal distributions with parameters reported in Table 1 where the value $A_{2} / A_{1}$ describes the relative amplitudes between the two skewed normals.

\section{COMPARISON WITH LITERATURE}

The models presented in this work predict a broad range of observables, many of which have already been compared to observations, either at lower redshifts, or of brighter objects than those considered in this paper. Below we provide a brief comparison with some of these observations, a more detailed discussion of many of these comparisons can be found in the original papers describing the models (Menci et al. 2012; Guo et al. 2011b; Lu et al. 2012).

\footnotetext{
${ }^{6}$ We do not consider the secondary peaks in the Menci models in this fit.
} 


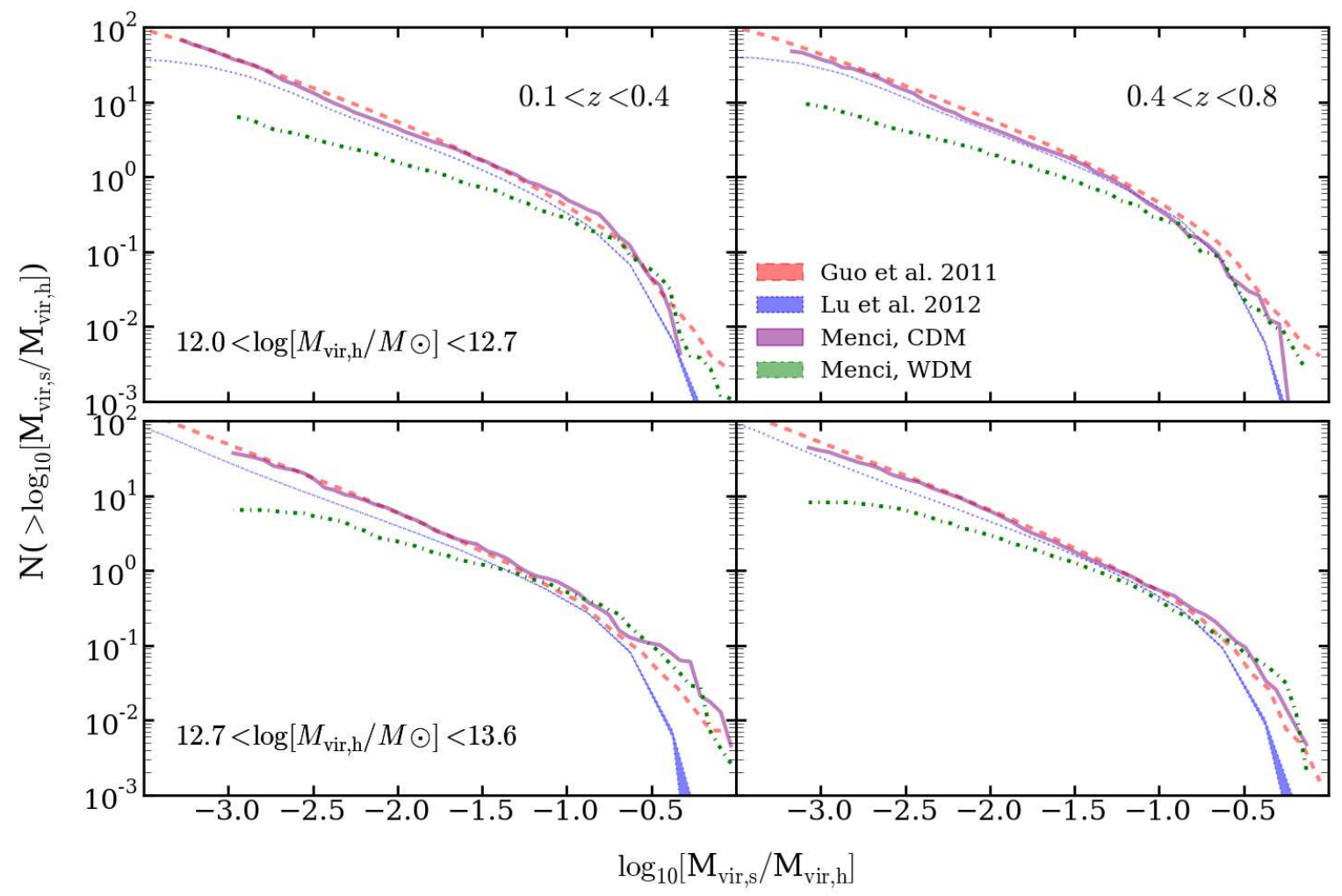

FIG. 3.- Comparison of the subhalo mass function at the time of subhalo accretion for the four models, for a range of masses representative of the host halos and subhalos in Figure 2 Note that these mass functions do not include the effects of tidal stripping by the main halo, nor are they selected to host luminous satellite galaxies, or host galaxies with the same stellar masses as the hosts in Figure 2

TABLE 1

Best fit PARAMETERS OF THE COLOR Distributions

\begin{tabular}{|c|c|c|c|c|c|}
\hline Host Properties & Dust & Guo et al. 2012 & Lu et al. 2012 & Menci, CDM & Menci, WDM \\
\hline & & $c_{o}, \sigma, a$ & $c_{o}, \sigma, a$ & $\begin{array}{l}c_{o}, \sigma, a, \\
A 2 / A 1, c_{o, 2}, \sigma_{2}, a_{2}\end{array}$ & $\begin{array}{l}c_{o}, \sigma, a, \\
A 2 / A 1, c_{o, 2}, \sigma_{2}, a_{2}\end{array}$ \\
\hline \multirow[t]{2}{*}{$10.5<\log \left[M^{*} / M_{\odot}\right]<11.0,0.1<\mathrm{z}<0.4$} & Yes & $1.7,0.5,8.8$ & $1.9,0.4,2.5$ & $\begin{array}{l}1.6,0.2,-5.0 \\
1.4,1.0,1.5,12.5\end{array}$ & $\begin{array}{l}1.4,1.0,9.3 \\
0.2,3.3,0.25,-1.9\end{array}$ \\
\hline & No & $2.0,0.3,1.5$ & $1.7,0.5,7.0$ & $1.3,0.3,1.4$ & $1.9,0.5,-4.0$ \\
\hline \multirow[t]{2}{*}{$10.5<\log \left[M^{*} / M_{\odot}\right]<11.0,0.4<\mathrm{z}<0.8$} & & $1.6,0.5,5.6$ & $1.8,0.3,2.4$ & $\begin{array}{l}1.4,0.3,-9.0 \\
3.1,2.9,0.9,2.5\end{array}$ & $\begin{array}{l}1.3,0.3,3.0 \\
1.5,2.1,0.5,1.6\end{array}$ \\
\hline & & $1.9,0.3,1.2$ & $1.6,0.4,4.5$ & $1.7,0.5,-5.4$ & $1.7,0.4,-4.4$ \\
\hline \multirow[t]{2}{*}{$11.0<\log \left[M^{*} / M_{\odot}\right]<11.5,0.1<\mathrm{z}<0.4$} & & $1.8,0.6,5.1$ & $1.2,0.5,2.7$ & $\begin{array}{l}1.5,0.24,-5.9 \\
4,2.6,0.5,0\end{array}$ & $\begin{array}{l}1.3,1.1,5.5 \\
0.3,3.3,0.3,-4.5\end{array}$ \\
\hline & & $2.5,0.3,-1.2$ & $1.8,0.5,4.3$ & $2.0,0.5,-4.4$ & $1.9,0.5,-4.2$ \\
\hline \multirow[t]{2}{*}{$11.0<\log \left[M^{*} / M_{\odot}\right]<11.5,0.4<\mathrm{z}<0.8$} & & $1.7,0.5,4.6$ & $1.9,0.5,2.9$ & $\begin{array}{l}1.3,0.25,0 \\
2.3,2.9,0.7,-2.6\end{array}$ & $\begin{array}{l}1.6,0.2,0 \\
1.3,2.0,0.5,1.7\end{array}$ \\
\hline & & $2.4,0.3,-1.3$ & $1.7,0.5,3.7$ & $1.7,0.5,-6.2$ & $1.7,0.4,-5.3$ \\
\hline
\end{tabular}




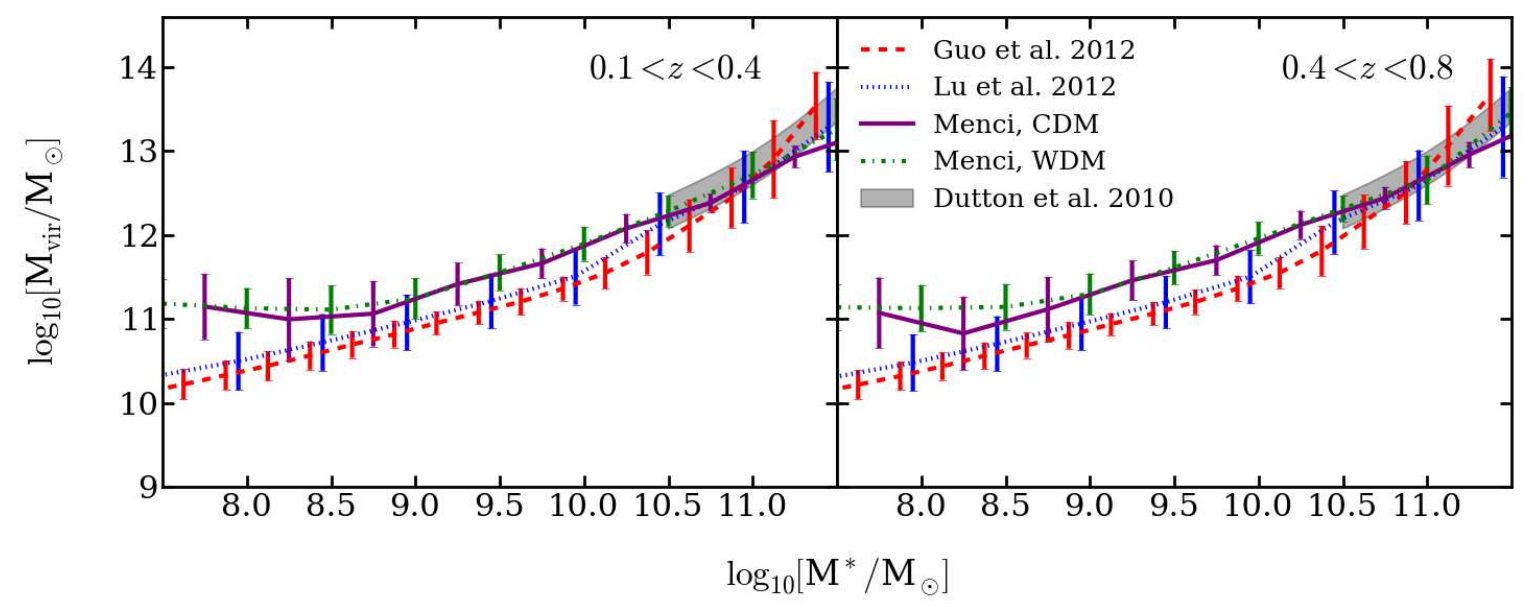

Fig. 4.- Comparison of the virial to stellar mass relationship for both host and satellite galaxies for the four models. The subhalo virial masses are taken at the time of accretion, and thus do not include the effects of tidal stripping. 


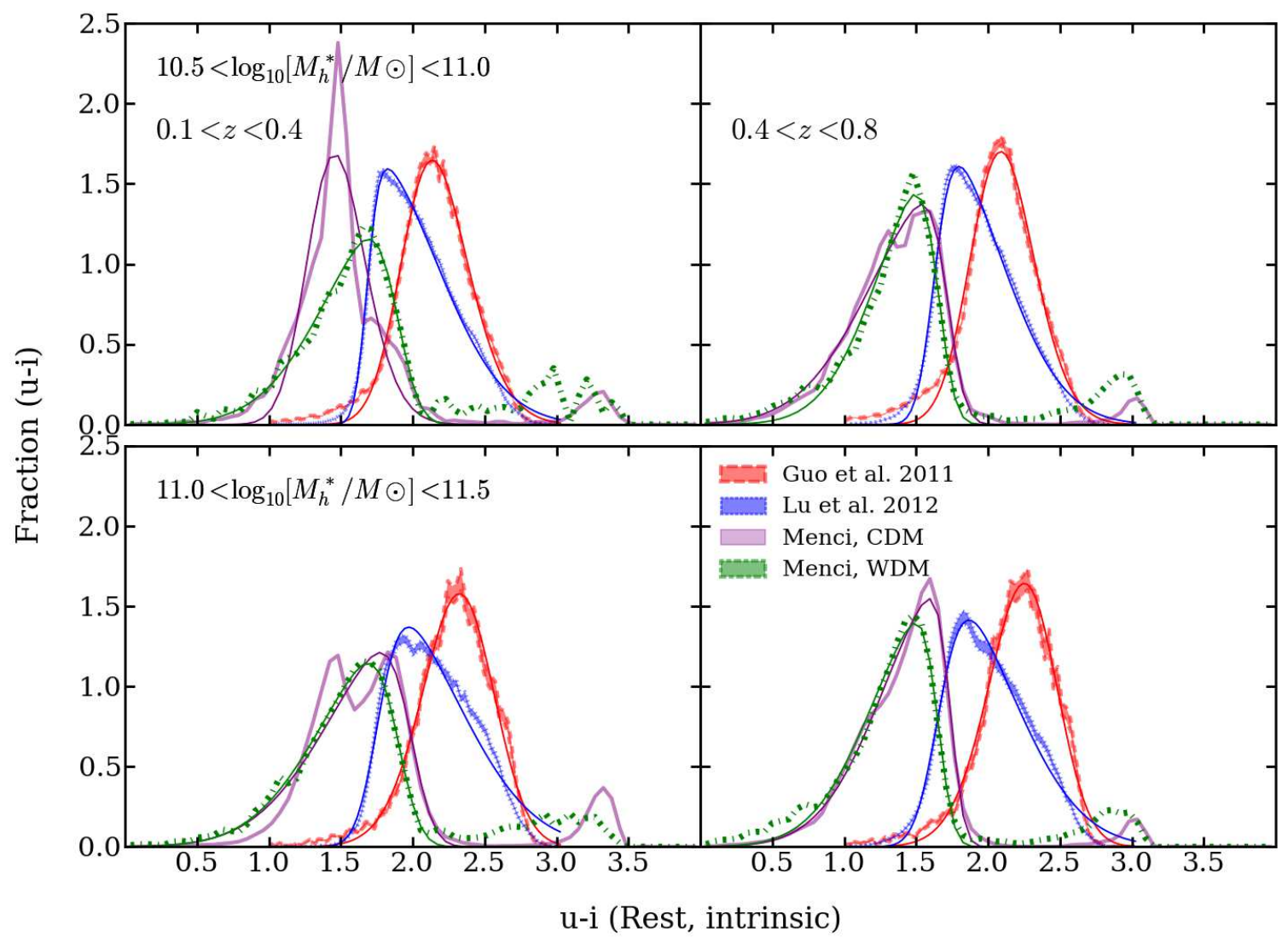

FIG. 5. - The normalized rest-frame distribution of colors, for the satellite galaxies plotted in Figure 2. The solid lines indicate the best fit skewed normal distributions defined by Equation 1 with fit parameters listed in Table 1

\subsection{Satellite luminosity function}

Studies of the satellite luminosity function typically focus on satellite galaxies at low redshifts with $z<0.1$, both in simulation and observations. As we have shown in Nierenberg et al. (2012), the COSMOS field can be used to study satellites at higher redshifts, and yields luminosity functions at low redshifts which are consistent with satellite luminosity functions from SDSS (e.g. Guo et al. 2011a; Liu et al. 2011; Lares et al. 2011; Strigari \& Wechsler 2012), and the Milky Way satellite luminosity function (Tollerud et al. 2008). All four of the models provide good fits to the satellite luminosity function of Milky Way mass hosts at low redshifts, by design. The extra information of redshift and host galaxy stellar mass provides additional constraints to these models. For instance, when comparing to bright field galaxies observed by Pérez-González et al. (2008), Guo et al. (2011b) found their model agreed well at low redshift, while becoming discrepant with observation by a redshift of 1 .

\subsection{Satellite Colors}

In Section 5.3 we showed that the three CDM models predicted significantly different distributions for the colors of faint satellite galaxies. Although we cannot directly test this prediction in this work, some comparisons can be made with measurements of field galaxies at low redshifts. In particular, Guo et al. (2011b) found that their model predicted colors matched observed SDSS colors well except for low masses $\left(\log _{10}\left[M^{*} / M_{\odot}\right]<9.5\right)$, which were redder in the simulation than in observations. Menci et al. (2012) found that down to an absolute magnitude of $M_{r}=-18$ (roughly a stellar mass of $\sim 9$ $\left.\log \left[M^{*} / M_{\odot}\right]\right)$, their color distribution agreed well with SDSS measurements from Baldry et al. (2004). They did not compare for fainter satellites.

The Lu and Guo semi-analytic models were not optimized to reproduce the observed galaxy color-magnitude relation at low redshifts, thus future implementations of these semi-analytic models which include this information may yield significantly different predictions for the color distribution of satellite galaxies.

Recently, Knobel et al. (2013) measured the red fraction of massive $\left(\log _{10}\left[M^{*} / M_{\odot}\right]>10\right)$ satellites between redshift 0.1 and 0.8 , and found no evidence for significant evolution over this time, indicating that these low redshift results may apply to higher redshifts. From a different point of view, Behroozi et al. (2012) used abundance matching techniques to infer that low stellar mass $\left(\log _{10}\left[M^{*} / M_{\odot}\right] \sim 9\right)$ field galaxies on average continue to form a significant fraction of their stars at redshift $\sim 1$. However, this result cannot be directly applied to 


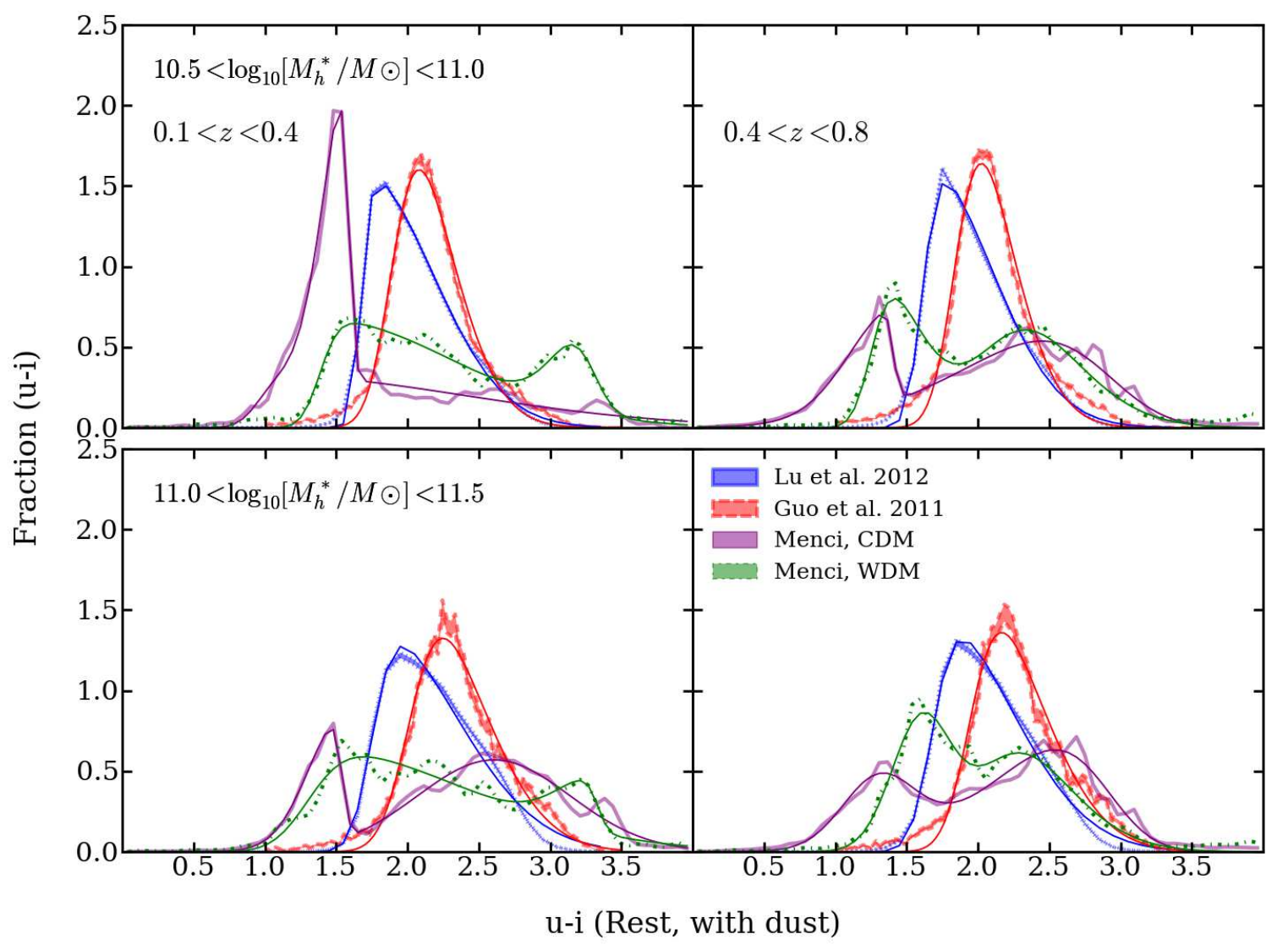

FIG. 6. - The normalized rest-frame distribution of colors, with dust extinction for the satellite galaxies plotted in Figure 2, along with the best fitting skewed normal distributions. The Menci models are fit with a sum of two skew normal distributions, to account for the prominent secondary peak that appears.

satellite galaxies, as star formation in satellite galaxies has been shown to be quenched relative to field galaxies at low redshifts (Pasquali et al. 2010; Geha et al. 2012; Kauffmann et al. 2004).

Future measurements of faint satellite colors since intermediate redshifts will provide interesting new constraints on these models, by helping to distinguish between suppression of the satellite luminosity function by environmental quenching and supernovae feedback, or the subhalo mass function at the low mass end.

\section{DISCUSSION AND CONCLUSION}

We have compared predictions from three current semianalytic models applied to CDM simulations and one semi-analytic model applied to a WDM simulation to observations of satellite galaxies in two redshift intervals and with two host mass bins. By comparing multiple models, we demonstrated that the effects of varying starformation prescriptions within semi-analytic models, and varying the underlying dark matter mass function have fundamentally different effects on the predicted host stellar mass dependence, and redshift evolution of the satellite luminosity function. In particular, the WDM model predicts a satellite luminosity function with much weaker host stellar mass dependence and redshift evolution than any of the CDM models.

We find that the WDM provides the best match to observation in all redshift and host mass intervals, most closely matching the host mass dependence and lack of redshift evolution in the data. This comparison highlights the importance of comparing models of satellite galaxy evolution to observations from a range of redshifts and stellar mass regimes, as a model that provides a close match to observation in one regime can perform more poorly than other models in a different regime.

This exploration of different CDM models suggests that current models generically have difficulty reproducing the mass and redshift dependence of the satellite luminosity function. However, future improvements to the semi-analytic models for star formation prescriptions may allow for improvements between the CDM predictions of the satellite luminosity function. In fact, as shown in the paper by Nierenberg et al. (2012) subhalo abundance matching techniques (Busha et al. 2011) can reconcile our observations with the CDM subhalo mass function. Although abundance matching is descriptive and not directly linked to known physical processes, it is possible that future semi-analytic models will be able to produce the stellar mass to halo mass relation as illustrated by this approach. 
Numerous studies have shown that abundance matching techniques can be used to map measured properties of star formation to simulated halo masses, and thus provide important constraints for semi-analytic models (e.g Behroozi et al. 2012; Reddick et al. 2012; Behroozi et al. 2010; Busha et al. 2011). The descriptive power of abundance matching is limited for low mass satellite galaxies by the resolution of dark matter simulations and the depth and redshift range of observations. Higher resolution simulations with varying dark matter power spectra, in conjunction with environmentally dependent abundance matching, can potentially provide very interesting constraints for the physical processes governing star formation in low mass halos in varying cosmologies.

Additional observational data are needed to further constrain the models and therefore help distinguish between whether the discrepancy between predicted and observed satellite luminosity function can be mitigated by either improved baryonic physics in CDM models or a WDM power spectrum. For example, we have shown in this work that the Lu CDM model which most closely matches the data predicts a significantly different color distribution for the satellite galaxies than the Menci WDM model, indicating that future observations of the colors of faint satellites will provide an important test of whether the luminosity function of satellites is sup- pressed primarily by baryonic processes or by a WDM mass function. We plan to carry out such measurement in the near future by exploiting the rich multicolor datasets publicly available in the HST legacy fields.

A.M.N. and T.T. acknowledge support from NSF through CAREER grant. T.T. acknowledges support from the Packard Foundations through a Packard Research Fellowship. We sincerely thank A. Benson, A. Peters, R. Wechsler and F. van den Bosch for useful comments and insightful conversations. This work was based on observations made with the NASA/ESA Hubble Space Telescope, and obtained from the Data Archive at the Space Telescope Science Institute, which is operated by the Association of Universities for Research in Astronomy, Inc., under NASA contract NAS 5-26555. These observations are associated with the COSMOS and GOODS projects. Part of the work presented in this paper was performed by AMN and TT while attending the program "First Galaxies and Faint Dwarfs: Clues to the Small Scale Structure of Cold Dark Matter" at the Kavli Institute of Theoretical Physics at the University of California Santa Barbara, supported in part by the National Science Foundation under Grant No. NSF PHY05-51164.

\section{REFERENCES}

Abazajian, K., Fuller, G. M., \& Patel, M. 2001, Phys. Rev. D, 64 023501

Abazajian, K. N., \& Kaplinghat, M. 2012, Phys. Rev. D, 86, 083511

Amara, A., Metcalf, R. B., Cox, T. J., \& Ostriker, J. P. 2006, MNRAS, 367, 1367

Azzalini, A. 1985, Scandinavian Journal of Statistics, 12, 171

Baldry, I. K., Glazebrook, K., Brinkmann, J., Ivezić, Ž., Lupton, R. H., Nichol, R. C., \& Szalay, A. S. 2004, ApJ, 600, 681

Behroozi, P., Wechsler, R., \& Wu, H.-Y. 2012, Astrophysics Source Code Library, 10008

Behroozi, P. S., Conroy, C., \& Wechsler, R. H. 2010, ApJ, 717, 379

Bell, E. F., McIntosh, D. H., Katz, N., \& Weinberg, M. D. 2003, ApJS, 149, 289

Benson, A. J., Farahi, A., Cole, S., Moustakas, L. A., Jenkins, A., Lovell, M., Kennedy, R., Helly, J., \& Frenk, C. 2013, MNRAS, 428, 1774

Benson, A. J., Frenk, C. S., Lacey, C. G., Baugh, C. M., \& Cole, S. 2002, MNRAS, 333, 177

Binney, J., \& Tremaine, S. 1987, Galactic dynamics

Bode, P., Ostriker, J. P., \& Turok, N. 2001, ApJ, 556, 93

Bond, J. R., Cole, S., Efstathiou, G., \& Kaiser, N. 1991, ApJ, 379, 440

Boyarsky, A., Lesgourgues, J., Ruchayskiy, O., \& Viel, M. 2009, Physical Review Letters, 102, 201304

Boylan-Kolchin, M., Bullock, J. S., \& Kaplinghat, M. 2012, MNRAS, 422, 1203

Boylan-Kolchin, M., Springel, V., White, S. D. M., Jenkins, A., \& Lemson, G. 2009, MNRAS, 398, 1150

Bruzual, G., \& Charlot, S. 2003, MNRAS, 344, 1000

Bullock, J. S., Kravtsov, A. V., \& Weinberg, D. H. 2000, ApJ, 539,517

Busha, M. T., Wechsler, R. H., Behroozi, P. S., Gerke, B. F., Klypin, A. A., \& Primack, J. R. 2011, ApJ, 743, 117

Carlberg, R. G., Grillmair, C. J., \& Hetherington, N. 2012, ApJ, 760,75

Chen, J. 2008, A\&A, 484, 347

Colín, P., Avila-Reese, V., \& Valenzuela, O. 2000, ApJ, 542, 622

Colombi, S., Dodelson, S., \& Widrow, L. M. 1996, ApJ, 458, 1

Conroy, C., \& Wechsler, R. H. 2009, ApJ, 696, 620
Croton, D. J., Springel, V., White, S. D. M., De Lucia, G., Frenk, C. S., Gao, L., Jenkins, A., Kauffmann, G., Navarro, J. F., \& Yoshida, N. 2006, MNRAS, 365, 11

Dalal, N., \& Kochanek, C. S. 2002, ApJ, 572, 25

De Lucia, G., \& Blaizot, J. 2007, MNRAS, 375, 2

de Vega, H. J., \& Sanchez, N. G. 2012, Phys. Rev. D, 85, 043517

Dolgov, A. D., \& Hansen, S. H. 2002, Astroparticle Physics, 16, 339

Doyle, M. T., Drinkwater, M. J., Rohde, D. J., Pimbblet, K. A., Read, M., Meyer, M. J., Zwaan, M. A., Ryan-Weber, E., Stevens, J., Koribalski, B. S., Webster, R. L., Staveley-Smith, L., Barnes, D. G., Howlett, M., Kilborn, V. A., Waugh, M., Pierce, M. J., Bhathal, R., de Blok, W. J. G., Disney, M. J., Ekers, R. D., Freeman, K. C., Garcia, D. A., Gibson, B. K., Harnett, J., Henning, P. A., Jerjen, H., Kesteven, M. J., Knezek, P. M., Mader, S., Marquarding, M., Minchin, R. F. O'Brien, J., Oosterloo, T., Price, R. M., Putman, M. E., Ryder, S. D., Sadler, E. M., Stewart, I. M., Stootman, F., \& Wright, A. E. 2005, MNRAS, 361, 34

Dunkley, J., Spergel, D. N., Komatsu, E., Hinshaw, G., Larson, D., Nolta, M. R., Odegard, N., Page, L., Bennett, C. L., Gold, B., Hill, R. S., Jarosik, N., Weiland, J. L., Halpern, M., Kogut, A., Limon, M., Meyer, S. S., Tucker, G. S., Wollack, E., \& Wright, E. L. 2009, ApJ, 701, 1804

Dutton, A. A., Conroy, C., van den Bosch, F. C., Prada, F., \& More, S. 2010, MNRAS, 407, 2

Fadely, R., \& Keeton, C. R. 2012, MNRAS, 419, 936

Garrison-Kimmel, S., Rocha, M., Boylan-Kolchin, M., Bullock, J., \& Lally, J. 2013, ArXiv e-prints

Geha, M., Blanton, M. R., Yan, R., \& Tinker, J. L. 2012, ApJ, 757,85

Gottloeber, S., Hoffman, Y., \& Yepes, G. 2010, ArXiv e-prints

Governato, F., Zolotov, A., Pontzen, A., Christensen, C., Oh, S. H., Brooks, A. M., Quinn, T., Shen, S., \& Wadsley, J. 2012, MNRAS, 422, 1231

Guo, Q., Cole, S., Eke, V., \& Frenk, C. 2011a, MNRAS, 1278

Guo, Q., White, S., Boylan-Kolchin, M., De Lucia, G., Kauffmann, G., Lemson, G., Li, C., Springel, V., \& Weinmann, S. 2011b, MNRAS, 413, 101 
Jarosik, N., Bennett, C. L., Dunkley, J., Gold, B., Greason, M. R., Halpern, M., Hill, R. S., Hinshaw, G., Kogut, A., Komatsu, E., Larson, D., Limon, M., Meyer, S. S., Nolta, M. R., Odegard, N., Page, L., Smith, K. M., Spergel, D. N., Tucker, G. S., Weiland, J. L., Wollack, E., \& Wright, E. L. 2011, ApJS, 192, 14

Kamada, A., Yoshida, N., Kohri, K., \& Takahashi, T. 2013, JCAP, 3,8

Kang, X., Macciò, A. V., \& Dutton, A. A. 2013, ApJ, 767, 22

Kauffmann, G., White, S. D. M., Heckman, T. M., Ménard, B., Brinchmann, J., Charlot, S., Tremonti, C., \& Brinkmann, J. 2004, MNRAS, 353, 713

Kaufmann, T., Bullock, J. S., Maller, A., \& Fang, T. 2008, in American Institute of Physics Conference Series, Vol. 1035, The Evolution of Galaxies Through the Neutral Hydrogen Window, ed. R. Minchin \& E. Momjian, 147-150

Keeton, C. R., \& Moustakas, L. A. 2009, ApJ, 699, 1720

Kennicutt, Jr., R. C. 1998, ApJ, 498, 541

Klypin, A., Kravtsov, A. V., Valenzuela, O., \& Prada, F. 1999, ApJ, 522, 82

Klypin, A. A., Trujillo-Gomez, S., \& Primack, J. 2011, ApJ, 740, 102

Knobel, C., Lilly, S. J., Kovač, K., Peng, Y., Bschorr, T. J., Carollo, C. M., Contini, T., Kneib, J.-P., Le Fevre, O., Mainieri, V., Renzini, A., Scodeggio, M., Zamorani, G., Bardelli, S., Bolzonella, M., Bongiorno, A., Caputi, K., Cucciati, O., de la Torre, S., de Ravel, L., Franzetti, P., Garilli, B., Iovino, A., Kampczyk, P., Lamareille, F., Le Borgne, J.-F., Le Brun, V., Maier, C., Mignoli, M., Pello, R., Perez Montero, E., Presotto, V., Silverman, J., Tanaka, M., Tasca, L., Tresse, L., Vergani, D., Zucca, E., Barnes, L., Bordoloi, R., Cappi, A., Cimatti, A., Coppa, G., Koekemoer, A. M., López-Sanjuan, C., McCracken, H. J., Moresco, M., Nair, P., Pozzetti, L., \& Welikala, N. 2013, ApJ, 769, 24

Komatsu, E., Dunkley, J., Nolta, M. R., Bennett, C. L., Gold, B., Hinshaw, G., Jarosik, N., Larson, D., Limon, M., Page, L. Spergel, D. N., Halpern, M., Hill, R. S., Kogut, A., Meyer, S. S., Tucker, G. S., Weiland, J. L., Wollack, E., \& Wright, E. L. 2009, ApJS, 180, 330

Komatsu, E., Smith, K. M., Dunkley, J., Bennett, C. L., Gold, B., Hinshaw, G., Jarosik, N., Larson, D., Nolta, M. R., Page, L., Spergel, D. N., Halpern, M., Hill, R. S., Kogut, A., Limon, M., Meyer, S. S., Odegard, N., Tucker, G. S., Weiland, J. L., Wollack, E., \& Wright, E. L. 2011, ApJS, 192, 18

Kravtsov, A. 2010, Advances in Astronomy, 2010

Kravtsov, A. V., Berlind, A. A., Wechsler, R. H., Klypin, A. A. Gottlöber, S., Allgood, B., \& Primack, J. R. 2004, ApJ, 609, 35

Kuhlen, M., Diemand, J., \& Madau, P. 2008, ApJ, 686, 262

Lares, M., Lambas, D. G., \& Domínguez, M. J. 2011, AJ, 142, 13

Liu, L., Gerke, B. F., Wechsler, R. H., Behroozi, P. S., \& Busha, M. T. 2011, ApJ, 733, 62

Lovell, M. R., Eke, V., Frenk, C. S., Gao, L., Jenkins, A., Theuns, T., Wang, J., White, S. D. M., Boyarsky, A., \& Ruchayskiy, O. 2012, MNRAS, 420, 2318

Lu, Y., Mo, H. J., Katz, N., \& Weinberg, M. D. 2012, MNRAS, 421, 1779

Lu, Y., Mo, H. J., Weinberg, M. D., \& Katz, N. 2011, MNRAS, 416, 1949

Macciò, A. V. 2010, in American Institute of Physics Conference Series, Vol. 1240, American Institute of Physics Conference Series, ed. V. P. Debattista \& C. C. Popescu, 355-358

Mao, S., \& Schneider, P. 1998, MNRAS, 295, 587
Menci, N., Fiore, F., \& Lamastra, A. 2012, MNRAS, 421, 2384

Metcalf, R. B., \& Madau, P. 2001, ApJ, 563, 9

Moore, B., Ghigna, S., Governato, F., Lake, G., Quinn, T., Stadel, J., \& Tozzi, P. 1999, ApJ, 524, L19

Newman, A. B., Ellis, R. S., Bundy, K., \& Treu, T. 2012, ApJ, 746,162

Nierenberg, A. M., Auger, M. W., Treu, T., Marshall, P. J., \& Fassnacht, C. D. 2011, ApJ, 731, 44

Nierenberg, A. M., Auger, M. W., Treu, T., Marshall, P. J., Fassnacht, C. D., \& Busha, M. T. 2012, ApJ, 752, 99

Olive, K. A., \& Turner, M. S. 1982, Phys. Rev. D, 25, 213

Papastergis, E., Martin, A. M., Giovanelli, R., \& Haynes, M. P. 2011, ApJ, 739, 38

Pasquali, A., Gallazzi, A., Fontanot, F., van den Bosch, F. C., De Lucia, G., Mo, H. J., \& Yang, X. 2010, MNRAS, 407, 937

Pérez-González, P. G., Rieke, G. H., Villar, V., Barro, G., Blaylock, M., Egami, E., Gallego, J., Gil de Paz, A., Pascual,

S., Zamorano, J., \& Donley, J. L. 2008, ApJ, 675, 234

Polisensky, E., \& Ricotti, M. 2011, Phys. Rev. D, 83, 043506

Porter, T. A., Johnson, R. P., \& Graham, P. W. 2011, ARA\&A, 49, 155

Purcell, C. W., \& Zentner, A. R. 2012, JCAP, 12, 7

Reddick, R. M., Wechsler, R. H., Tinker, J. L., \& Behroozi, P. S. 2012, ArXiv e-prints

Shi, X., \& Fuller, G. M. 1999, Physical Review Letters, 82, 2832

Smith, R. E., \& Markovic, K. 2011, Phys. Rev. D, 84, 063507

Somerville, R. S. 2002, ApJ, 572, L23

Spergel, D. N., Verde, L., Peiris, H. V., Komatsu, E., Nolta, M. R., Bennett, C. L., Halpern, M., Hinshaw, G., Jarosik, N., Kogut, A., Limon, M., Meyer, S. S., Page, L., Tucker, G. S., Weiland, J. L., Wollack, E., \& Wright, E. L. 2003, ApJS, 148, 175

Springel, V. 2010, ARA\&A, 48, 391

Springel, V., Frenk, C. S., \& White, S. D. M. 2006, Nature, 440, 1137

Springel, V., White, S. D. M., Jenkins, A., Frenk, C. S., Yoshida, N., Gao, L., Navarro, J., Thacker, R., Croton, D., Helly, J., Peacock, J. A., Cole, S., Thomas, P., Couchman, H., Evrard, A., Colberg, J., \& Pearce, F. 2005, Nature, 435, 629

Steffen, F. D. 2006, JCAP, 9,

Strigari, L. E. 2012, ArXiv e-prints

Strigari, L. E., Bullock, J. S., Kaplinghat, M., Diemand, J., Kuhlen, M., \& Madau, P. 2007, ApJ, 669, 676

Strigari, L. E., \& Wechsler, R. H. 2012, ApJ, 749, 75

Teyssier, R., Pontzen, A., Dubois, Y., \& Read, J. I. 2013, MNRAS, 493

Tollerud, E. J., Bullock, J. S., Strigari, L. E., \& Willman, B. 2008, ApJ, 688, 277

Treu, T. 2010, ARA\&A, 48, 87

Tully, R. B., \& Fisher, J. R. 1977, A\&A, 54, 661

Vegetti, S., Lagattuta, D. J., McKean, J. P., Auger, M. W. Fassnacht, C. D., \& Koopmans, L. V. E. 2012, Nature, 481, 341

Viel, M., Bolton, J. S., \& Haehnelt, M. G. 2009, MNRAS, 399 L39

Wang, J., Frenk, C. S., Navarro, J. F., Gao, L., \& Sawala, T. 2012, MNRAS, 424, 2715

Wang, W., \& White, S. D. M. 2012, MNRAS, 424, 2574

Watson, D. F., Berlind, A. A., McBride, C. K., Hogg, D. W., \& Jiang, T. 2012, ApJ, 749, 83 